\title{
ON THE FRATTINI SUBGROUPS OF CERTAIN GENERALIZED FREE PRODUCTS OF GROUPS ${ }^{1}$
}

\author{
C. Y. TANG
}

\begin{abstract}
Let $G=\left(\prod_{i \in I}^{*} A_{i}\right)_{H}$ be the generalized free product of the groups $A_{i}$ amalgamating the subgroup $H$. We show that if $G$ is residually finite and the groups $A_{i}$ have compatible $H$-filters then the Frattini subgroup $\Phi(G)$ is contained in the maximal $G$-normal subgroup in $H$. If the groups $A_{i}$ are free and $H$ is finitely generated of infinite index in one $A_{i}$ then $\Phi(G)=1$. We also show that if $H$ is simple then $\Phi(G)=1$ or $H^{G}$.
\end{abstract}

1. The purpose of this paper is to continue our effort to answer the questions raised by Higman and Neumann [5]: whether the Frattini subgroup of a generalized free product can be larger than the amalgamated subgroup and whether such groups necessarily have maximal subgroups. In [8] Whittemore answered these questions for generalized free products of finitely many free groups amalgamating a cyclic subgroup and for generalized free products of finitely many finitely generated abelian groups. In [2] Djoković and Tang showed that if $G$ is the generalized free product amalgamating the subgroup $H$ which satisfies the minimal condition on subgroups then the Frattini subgroup $\Phi(G)$ is contained in the maximal $G$-normal subgroup contained in $H$. In this paper we shall extend the results in [2] and [8]. In particular, we show that if $G=\left(\Pi^{*} A_{i}\right)_{H}$ is residually finite and the $A_{i}$ have compatible $H$-filters then $\Phi(G)$ is contained in the maximal $G$-normal subgroup contained in $H$. In the case of $G=\left(\prod_{i \in I}^{*} A_{i}\right)_{H}$ where each $A_{i}$ is free and $H$ is finitely generated then $\Phi(G)=1$ if $H$ is of infinite index in one of the $A_{i}$. We also show that if the amalgamated subgroup is simple then $\Phi(G)=1$ or $H^{G}$.

The notation used is in general standard:

$G=(A * B)_{H I}$ denotes the generalized free product of $A$ and $B$ amalgamating the subgroup $H$.

A subgroup $N$ is said to be $G$-normal if $N \triangleleft G$.

$G \backslash H$ denotes the set of elements of $G$ not contained in $H$.

$\left\langle a_{1}, \cdots, a_{n}\right\rangle$ denotes the group generated by the elements $a_{1}, \cdots, a_{n}$.

Received by the editors February 9, 1971 and, in revised form, February 18, 1972. AMS (MOS) subject classifications (1970). Primary 20E20, 20E30; Secondary 20F30.

Key words and phrases. Frattini subgroup, free group, simple group, generalized free product, amalgamated subgroup, residually finite, $H$-filter, $G$-normal, identical relation.

${ }^{1}$ This work was partly supported by a grant from the Canadian National Research Council No. A-4064.

(C) American Mathematical Society 1973 
Let $x \in G=\left(\prod_{i \in I}^{*} A_{i}\right)_{H}$. Then init $x$ and end $x$ shall respectively denote an initial letter and an end letter of $x$ when $x$ is expressed as $a_{\alpha_{1}} a_{\alpha_{2}} \cdots a_{\alpha_{n}}$ where $a_{\alpha_{i}} \in A_{j}$ and no two consecutive $a_{\alpha_{i}}$ belong to the same $A_{j}$. We note that for a given $x$ both init $x$ and end $x$ are not uniquely defined. However any two init $x$ belong to the same $A_{i}$ for a given $x$. The same is true for end $x$. Also $l(x)$ shall denote the length of $x$ in $G$.

The following terms and notation are the same as Baumslag [1]:

$\left\{S_{i} ; i \in I\right\}$ is a filter of $G$ if each $S_{i}$ is a normal subgroup of finite index in $G$ and $\bigcap_{I} S_{i}=1$.

$\left\{S_{i} ; i \in I\right\}$ is called an $H$-filter of $G$ where $H$ is a subgroup of $G$ if it is a filter of $G$ and $\bigcap_{I} S_{i} H=H$.

If $G=(A * B)_{H}$ and $\left\{A_{i} ; i \in I\right\},\left\{B_{i} ; i \in I\right\}$ are filters of $A$ and $B$ respectively then they are said to be compatible with respect to $H$ if $A_{i} \cap H=$ $B_{i} \cap H$ for $i \in I$.

The following result from [2] will be needed:

THEOREM 1.1. Let $G=(A * B)_{H}$. If $H$ satisfies the minimal condition on subgroups then $\Phi(G)$ is contained in the maximal G-normal subgroup contained in $H$.

Remark. Throughout the paper $G=(A * B)_{H}$ shall be assumed to be a nontrivial generalized free product. Although we prove our results for $G=(A * B)_{H}$ only, it is not difficult to extend the results to $G=\left(\prod_{i \in I}^{*} A_{i}\right)_{H}$ for arbitrary $I$.

2. We shall first prove a theorem which we need in $\S 3$.

THEOREM 2.1. Let $G=(A * B)_{I r}$. If $\Phi(G) \cap H=1$ then $\Phi(G)=1$.

Proof. Let $x \in \Phi(G)$ and $h \in H$. If $h^{x} \in H$ then $[x, h] \in \Phi(G) \cap H=1$. Thus $x$ and $h$ commute whenever $h^{x} \in H$. Suppose $\Phi(G) \neq 1$. Since $\Phi(G) \cap H=1$ and $\Phi(G) \triangleleft G$, it is not difficult to see that there exists an element $c \in \Phi(G)$ such that init $c \in B \backslash H$ and end $c \in A \backslash H$; for example, if $x \in \Phi(G)$ is of the form $a_{1} b_{1} \cdots a_{n} b_{n} a_{n+1}$ where $a_{i}, b_{i} \notin H$, then for $b \in B \backslash H, c=x^{b} \cdot x \in \Phi(G)$ will be of the required form. Clearly the order of $c$ is infinite, whence $c^{2} \neq 1$. Let $X=\left\langle A^{c^{2}}, B\right\rangle$. We shall show that $c \notin X$. Suppose $c \in X$. Then $c$ would be of the form:

$$
c=b_{0} c^{-2} a_{1} c^{2} b_{1} c^{-2} a_{2} c^{2} b_{2} \cdots c^{-2} a_{n} c^{2} b_{n}
$$

where $a_{i} \in A$ and $b_{i} \in B$. Without loss of generality we can assume $n$ to be minimal. We shall show that equation (1) cannot hold.

Case 1. $a_{1} \in H$ and $a_{1}^{c} \in H$. As noted earlier this means $a_{1}^{c}=a_{1} \in H$. Thus $b_{1} \notin H$, for otherwise $n$ would not be minimal. Also $a_{2}^{c} \notin H$; for if $a_{2}^{c} \in H$ then we must have $a_{2} \in H$ since init $c \in B \backslash H$. But then $\left[c, a_{2}\right]=1$ 
which would violate the minimal choice of $n$. Using the same argument we can assume $b_{i}^{c^{-1}} \notin H$ for $i=2, \cdots, n-1$ and $a_{i}^{c} \notin H$ for $i=2, \cdots, n$. Now it is easy to see that for $n>1$ the length of the R.H.S. (right hand side) of (1) is greater than $l(c)$ since init $c \in B \backslash H$ and end $c \in A \backslash H$. Hence (1) cannot hold. On the other hand if $n \leqq 1$, then the length of the R.H.S. of (1) is 0 or 1 . But by the choice of $c, l(c)>1$. Hence again (1) cannot hold.

Case 2. $a_{1} \in H$ and $a_{1}^{c} \notin H$. As in Case 1 , because of the minimal choice of $n, b_{i}^{c^{-1}} \notin H$ for $i=1, \cdots, n-1$ and $a_{i}^{c} \notin H$ for $i=2, \cdots, n$ if $n \geqq 2$. It is not difficult to see that for $n \geqq 1$ the length of the R.H.S. of (1) is always greater than $l(c)$. On the other hand if $n=0$ then the length of the R.H.S. of (1) is always less than $l(c)$. Hence (1) cannot hold.

Case 3. $a_{1} \in A \backslash H$. Since init $c \in B \backslash H$, it follows that $a_{1}^{c} \notin H$. Applying the same argument for Case 2 we conclude that (1) cannot hold.

Thus in all possible cases the equation (1) cannot be true. Therefore $c \notin X$, whence $X \subsetneq G$. On the other hand $G=\langle X, c\rangle$. This implies that $c \notin \Phi(G)$ contradicting the assumption that $c \in \Phi(G)$. This contradiction comes from the assumption that $\Phi(G) \neq 1$. Hence $\Phi(G)=1$.

Since $\Phi(G) \cap H$ is normal in $H$ we have the following corollary:

CoRollary 2.2. Let $G=(A * B)_{H}$. If $H$ is a simple group then $\Phi(G)=1$ or $H^{G}$.

3. We shall now proceed to show that if $G=(A * B)_{I I}$ is residually finite and $A$ and $B$ have compatible $H$-filters then $\Phi(G)$ is contained in the maximal $G$-normal subgroup contained in $H$.

LEMMA 3.1. Let $G=(A * B)_{H}$. Let $S$ be a subgroup of $A$ and $T=S^{G}$, the normal closure of $S$ in $G$. Then $G / T=(\bar{A} * \bar{B})_{H}$ where $\bar{A}=A T / T$, $\bar{B}=B T / T$ and $\bar{H}=H T / T$.

Proof. Let $\bar{G}=(\bar{A} * \bar{B})_{H}$. Let $\theta_{A}: A \rightarrow \bar{A}$ and $\theta_{B}: B \rightarrow \bar{B}$ be respectively the restrictions of the canonical homomorphism from $G$ onto $G / T$. Then by a well-known theorem of B. H. Neumann $\left[7\right.$, p. 505] $0_{A}$ and $\theta_{B}$ can be extended to a homomorphism $\theta$ from $G$ onto $\bar{G}$. Now it is not difficult to show that $\operatorname{ker} \theta$ is $T$. Thus $G / T$ is not only isomorphic but actually equal to $\bar{G}$.

LeMmA 3.2. Let $G=(A * B)_{I I}$. If $U \triangleleft A$ and $V \triangleleft B$ such that $U \cap H=$ $V \cap H$ then $U^{G} \cap A=U$ and $V^{G} \cap B=V$.

Proof. Clearly $U \subseteq U^{G} \cap A$. Let $x \in U^{(i \cap} \cap A$. Then $x=u^{g}$ for some $u \in U$ and $g \in G$. Let $g=a_{1} b_{1} \cdots a_{n} b_{n}$ where $a_{1} \in A, a_{i} \in A \backslash H$ for $i=2, \cdots$, $n$ and $b_{n} \in B, b_{i} \in B \backslash H$ for $i=1, \cdots, n-1$. Then

$$
x=b_{n}^{-1} a_{n}^{-1} \cdots b_{1}^{-1} a_{1}^{-1} u a_{1} b_{1} \cdots a_{n} b_{n} .
$$


Since $x \in A$ we have $l(x) \leqq 1$. We shall show that $x \in U$ by induction on $n$. Let $n=1$. If $u^{a_{1}} \in A \backslash H$ then for (2) to hold we must have $b_{1} \in H$, for otherwise $l\left(x^{g}\right)=3$. Now $U \triangleleft A$. Therefore $x=u^{g} \in U$. Hence we need only consider the case when $u^{a_{1}} \in H$. But this implies that $u^{a_{1}} \in U \cap H=V \cap H$.

Case 1. $u^{a_{1} b_{1}} \in H$. Since $u^{a_{1}} \in V$ and $V \triangleleft B$ we have $u^{a_{1} b_{1}} \in V \cap H \subseteq U$. Hence $x=u^{g} \in U$.

Case 2. $u^{a_{1} b_{1}} \notin H$. Since $u^{a_{1}} \in H$ we must have $u^{a_{1} b_{1}} \in B \backslash H$. But this contradicts the assumption that $u^{g}=x \in A$. Hence this case cannot occur.

Let $n>1$. Let $g_{1}=a_{1} b_{1} \cdots a_{n-1} b_{n-1}$. If $l\left(u^{g_{1}}\right)>1$, then since $b_{n-1} \in B \backslash H$ and $a_{n} \in A \backslash H$ it follows that $l\left(u^{g}\right)>1$ whence (2) cannot hold. Therefore $l\left(u^{g_{1}}\right) \leqq 1$. Now $b_{n-1} \in B$ implies that $u^{g_{1}} \in B$. If $u^{g_{1}} \in B \backslash H$ then since $a_{n} \in$ $A \backslash H$ we have $l\left(u^{g_{1}}\right)>1$. Thus $u^{g_{1}} \in H$ whence $u^{g_{1}} \in U^{G} \cap H \subseteq U^{G} \cap A$. Hence by induction $u^{g_{1}} \in U \cap A$. Writing $u^{g_{1}}=u_{1} \in U$ we have $u_{1}^{a_{n} b_{n}} \in U$. Hence $x=u_{1}^{a_{n} b_{n}} \in U$ and $U^{G} \cap A \subseteq U$.

THEOREM 3.3. Let $G=(A * B)_{H}$ be residually finite. If $A$ and $B$ have compatible $H$-filters and $H$ contains no nontrivial $G$-normal subgroup, then $\Phi(G)=1$.

Proof. Let $\left\{U_{i} ; i \in I\right\}$ and $\left\{V_{i} ; i \in I\right\}$ be compatible $H$-filters of $A$ and $B$ respectively. Let $N_{i}=U_{i}^{G}$ and $G_{i}=G / N_{i}$. By Lemma 3.1, $G_{i}=\left(A_{i} * B_{i}\right)_{H_{i}}$ where $A_{i}=A N_{i} / N_{i}, B_{i}=B N_{i} / N_{i}$ and $H_{i}=H N_{i} / N_{i}$. It is not difficult to show that the $H$-filters can be chosen so that each $G_{i}$ is a nontrivial generalized free product. Now $A / U_{i}$ is finite, whence $H_{i}$ is finite. Let $M_{i}$ be the maximal $G_{i}$-normal subgroup contained in $H_{i}$. Then by Theorem 1.1, $\Phi\left(G_{i}\right) \subseteq M_{i}$. If $L_{i}$ is the set of preimages of $M_{i}$ in $G$ then $\Phi(G) \subseteq L_{i}$, whence $\Phi(G) \subseteq L$, where $L=\bigcap_{I} L_{i}$.

Now $L_{i} \subseteq N_{i} H$. Thus $L_{i} \cap A \subseteq N_{i} H \cap A \subseteq\left(N_{i} \cap A\right) H$. Because of the compatibility of the $H$-filters, $U_{i} \cap H=V_{i} \cap H$. Therefore, by Lemma 3.2, $N_{i} \cap A=U_{i}$, whence $L_{i} \cap A \subseteq U_{i} H$. Since $\left\{U_{i} ; i \in I\right\}$ is an $H$-filter, we have $\bigcap_{I} U_{i} H=H$. Hence,

$$
L \cap A \subseteq \bigcap_{I}\left(L_{i} \cap A\right) \subseteq \bigcap_{I} U_{i} H=H .
$$

Let $x \in \Phi(G) \cap H$ and $a \in A$. Since $L \triangleleft G$, we have $x^{a} \in L$, whence $x^{a} \in L \cap A \subseteq H$. But $x^{a} \in \Phi(G)$. Therefore $x^{a} \in \Phi(G) \cap H$. It follows that $\Phi(G) \cap H \triangleleft A$. In the same way we can show that $\Phi(G) \cap H \triangleleft B$. Hence $\Phi(G) \cap H \triangleleft G$. But $H$ contains no nontrivial $G$-normal subgroup. Therefore $\Phi(G) \cap H=1$, whence by Theorem $2.1, \Phi(G)=1$.

CoRollary 3.4. Let $G=(A * B)_{H}$ be residually finite. If $A$ and $B$ have compatible H-filters then $\Phi(G)$ is contained in the maximal G-normal subgroup contained in $H$. 
In [4] Gregorac showed that if $G=(A * B)_{H}$ is residually finite and $A$ and $B$ each satisfies a suitable identical relation then $A$ and $B$ have compatible $H$-filters. In fact the suitable identical relation required is a nontrivial identical relation which is not satisfied by the infinite dihedral group. Thus applying Theorem 2 [4] and Corollary 3.4, we have:

THEOREM 3.5. Let $G=(A * B)_{I I}$ be residually finite. Then $\Phi(G)$ is contained in the maximal $G$-normal subgroup contained in $H$ if one of the following conditions is satisfied:

(i) $A$ and $B$ each satisfies a nontrivial identical relation which is not satisfied by the infinite dihedral group;

(ii) $A$ and $B$ each satisfies a nontrivial identical relation and both $[A: H]$ and $[B: H]$ are greater than 2 ;

(iii) $[A: H]=[B: H]=2$.

Proof. (i) and (ii) are direct consequences of Theorem 2 [4] and Corollary 3.4. In the case of (iii) we note that $H \triangleleft G$. Thus, if $\left\{S_{i} ; i \in I\right\}$ is a filter for $G$, then $U_{i}=S_{i} \cap H$ is normal and of finite index in both $A$ and $B$. Hence $\left\{U_{i} ; i \in I\right\}$ is an $H$-filter for both $A$ and $B$.

We can apply Theorem 3.5 to various results on residual finiteness of generalized free products. For example, by Theorem 5 of Baumslag [1] and Theorem 6 of Dyer [3], we have the following results:

THEOREM 3.6. Let $G=(A * B)_{I I}$ where $A$ and $B$ are finitely generated torsion-free nilpotent groups and $H$ is closed in both $A$ and $B$. Then $\Phi(G)$ is contained in the maximal G-normal subgroup contained in $H$.

THEOREM 3.7. Let $G=(A * B)_{I I}$ where $A, B \in \mathscr{F} \cdot \mathscr{A}(\mathscr{F}$ being the class of finite groups and $\mathscr{A}$ the class of finitely generated abelian groups). Then $\Phi(G)$ is contained in the maximal G-normal subgroup contained in $H$.

4. In the preceding section results on the Frattini subgroups of residually finite generalized free products are obtained by applying Theorem 3.5. Unfortunately Theorem 3.5 requires $A$ and $B$ each to satisfy a nontrivial identical relation. Thus, in particular, if $A$ and $B$ are free groups we cannot make use of this theorem. In this section we shall derive some results in this direction.

LEMMA 4.1. Let $F$ be a free group and $H$ be a subgroup of $F$ such that for all $x \in F$ there exists $1 \neq h \in H$ and that $h^{x} \in H$. Then every nontrivial normal subgroup of $F$ intersects $H$ nontrivially.

Proof. Let $N$ be a nontrivial normal subgroup of $F$. Suppose $N \cap H=1$. Let $1 \neq x \in N$. Then there exists $1 \neq h \in H$ such that $h^{x} \in H$. This implies that $[h, x] \in H$. But $N \triangleleft F$. It follows that $[h, x] \in N \cap H=1$, whence $h$ 
and $x$ commute. Now $F$ is free. The centralizer of every nontrivial element of $F$ is cyclic. This implies that $h$ and $x$ belong to the same cyclic group. Thus $\langle h\rangle \cap\langle x\rangle \neq 1$, whence $H \cap N \neq 1$ contradicting the initial assumption.

LEMMA 4.2. Let $F$ be a free group and $H$ be a finitely generated subgroup of $F$ such that for all $x \in F$ w'e have $H \cap H^{x} \neq 1$. Then $H$ is of finite index in $F$.

Proof. $H \cap H^{x} \neq 1$ for $x \in F$ implies that there exists $1 \neq h \in H$ such that $h^{x} \in H$. Thus if $N$ is any nontrivial normal subgroup of $F$ then, by Lemma $4.1, N \cap H \neq 1$. Now $H$ is finitely generated, whence by Theorem 3 [6], $H$ is of finite index in $F$.

TheOREM 4.3. Let $G=(A * B)_{I I}$ where $A$ and $B$ are free groups and $H$ is finitely generated such that at least one of $[A: H]$ and $[B: H]$ is infinite. Then $\Phi(G)=1$.

Proof. We shall assume $[A: H]$ to be infinite. Suppose there does not exist $x \in A$ such that $H \cap H^{x}=1$. Then, by Lemma 4.2, $[A: H]$ is finite. Hence there exists $x \in A$ such that $H \cap H^{x}=1$. Thus by the Proposition $2.3[8], \Phi(G)=1$.

\section{REFERENCES}

1. G. Baumslag, On the residual finiteness of generalized free products of nilpotent groups, Trans. Amer. Math. Soc. 106 (1963), 193-209. MR 26 \#2489.

2. D. Ž. Djoković and C. Y. Tang, On the Frattini subgroup of the generalized free product with amalgamation, Proc. Amer. Math. Soc. 32 (1972), 21-23.

3. J. L. Dyer, On the residual finiteness of generalized free products, Trans. Amer. Math. Soc. 133 (1968), 131-143. MR 38 \#5930.

4. R. J. Gregorac, On residually finite generalized free products, Proc. Amer. Math. Soc. 24 (1970), 553-555. MR 41 \#5498.

5. G. Higman and B. H. Neumann, On two questions of Itô, J. London Math. Soc. 29 (1954), 84-88. MR 15, 286.

6. A. Karrass and D. Solitar, On finitely generated subgroups of a free group. Proc. Amer. Math. Soc. 22 (1969), 209-213. MR 39 \#6961.

7. B. H. Neumann, An essay on free products with amalgamations, Philos. Trans. Roy. Soc. London Ser. A 246 (1954), 503-544. MR 16, 10.

8. A. Whittemore, On the Frattini subgroup, Trans. Amer. Math. Soc. 141 (1969), 323-333. MR 39 \#6993.

Department of Mathematics, University of Waterloo, Waterloo, Ontario, CANADa

Department of Mathematics, National Tsing Hua University, Hsinchu, TAIWAN, Republic OF CHina 